\title{
A high magnification UV lens for high temperature optical strain measurements
}

Cite as: Rev. Sci. Instrum. 90, 045117 (2019); https://doi.org/10.1063/1.5081899

Submitted: 17 November 2018 . Accepted: 28 March 2019 . Published Online: 17 April 2019

Robert S. Hansen, Trevor J. Bird (D), Ren Voie, Katharine Z. Burn, and Ryan B. Berke

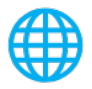

\section{ARTICLES YOU MAY BE INTERESTED IN}

A scalable arbitrary waveform generator for atomic physics experiments based on fieldprogrammable gate array technology

Review of Scientific Instruments 90, 043101 (2019); https://doi.org/10.1063/1.5051124

Non-destructive evaluation of tomato based on optical scattering

Review of Scientific Instruments 90, 043102 (2019); https://doi.org/10.1063/1.5082869

High-pressure laser floating zone furnace

Review of Scientific Instruments 90, 043906 (2019); https://doi.org/10.1063/1.5085327

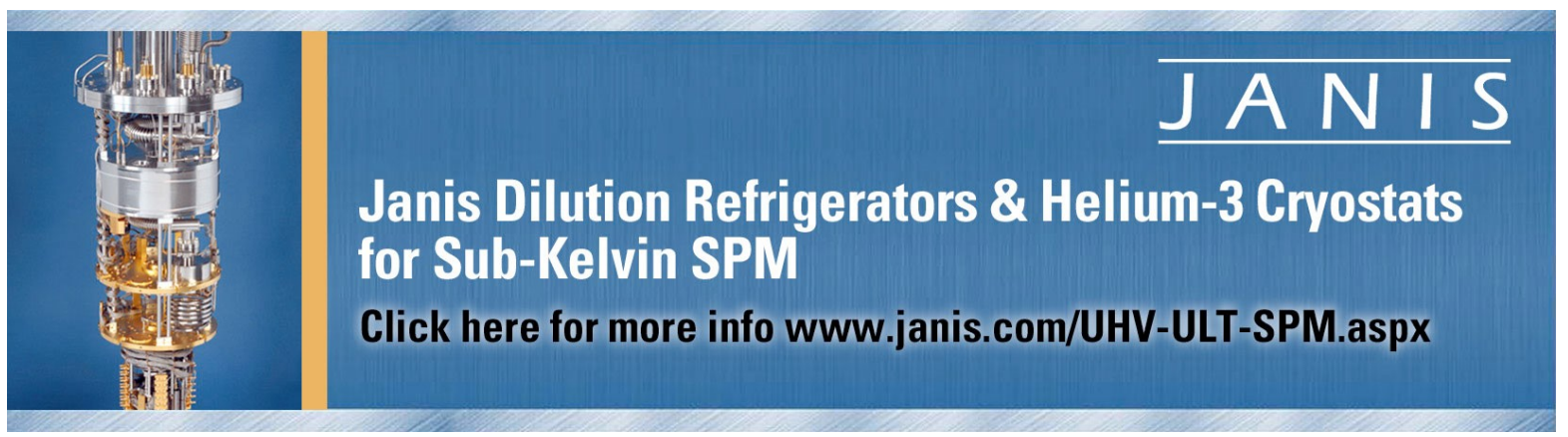




\title{
A high magnification UV lens for high temperature optical strain measurements
}

\author{
Cite as: Rev. Sci. Instrum. 90, 045117 (2019); doi: 10.1063/1.5081899 \\ Submitted: 17 November 2018 - Accepted: 28 March 2019 • \\ Published Online: 17 April 2019
}

Robert S. Hansen, Trevor J. Bird, (D) Ren Voie, Katharine Z. Burn, and Ryan B. Berke ${ }^{\text {a) }}$

AFFILIATIONS

Department of Mechanical and Aerospace Engineering, Utah State University, 4130 Old Main Hill, Logan, Utah 84322-4130, USA

\author{
a) Author to whom correspondence should be addressed: ryan.berke@usu.edu
}

\begin{abstract}
Digital Image Correlation (DIC) measures full-field strains by tracking displacements of a specimen using images taken before and after deformation. At high temperatures, materials emit light in the form of blackbody radiation, which can interfere with DIC images. To screen out that light, DIC has been recently adapted by using ultraviolet (UV) range cameras, lenses, and filters. Before now, UV-DIC had been demonstrated at the centimeter scale using commercially available UV lenses and filters. Commercial high-magnification lenses using visible light have also been used for DIC. However, there is currently no commercially available high-magnification lens that will allow images to be taken (a) in the UV range, (b) at a submillimeter scale, and (c) from a relatively long working distance separating a specimen inside a test chamber and a camera outside the chamber. In this work, a custom UV high-magnification lens is demonstrated to perform highmagnification, high-temperature DIC measurements. To demonstrate the capabilities of this lens, a series of thermo-mechanical tests was run on a stainless-steel ring specimen. Two UV cameras performed simultaneous measurements: one at lower magnification using a commercial UV lens, and one with the custom high-magnification UV lens. At room temperature, the custom lens produces sufficiently bright images to perform DIC, while at high temperature (demonstrated to $900{ }^{\circ} \mathrm{C}$ ) the images retained sufficient contrast while avoiding oversaturation. The lens can detect submillimeter rigid motion and tensile strains from long working distances and high magnification. These tests show that the custom lens is suitable for use in high-magnification UV-DIC measurements.
\end{abstract}

Published under license by AIP Publishing. https://doi.org/10.1063/1.5081899

\section{INTRODUCTION}

Digital Image Correlation (DIC) is a non-contact method used to produce full-field displacement and strain measurements of a specimen surface. It is a popular strain measurement in a variety of experimental applications because it (i) is a noncontact measurement, ${ }^{1}$ (ii) is easily adapted to different length scales, ${ }^{2}$ and (iii) provides strain distributions across an entire surface rather than a single point. ${ }^{3}$ These advantages make DIC an ideal candidate for measuring heterogeneous strain in high-temperature and highmagnification situations. DIC has been demonstrated at a wide variety of length scales, ranging from large scale to microscale ${ }^{4}$ and even nanoscale, ${ }^{5}$ with the use of scanning electron microscopes. Smallscale measurements are especially useful in materials characterization, ${ }^{6}$ when features such as grains or small platelets and precipitates in a specimen are particularly important.'

To compute displacements and strains using DIC, images must be taken before and after deformation. There are two main approaches to performing DIC: subset-based local, and finiteelement-based global digital image correlation. The local DIC algorithm divides a region of interest into subsets surrounding a single pixel and tracks the motion of each subset using a correlation algorithm, resulting in a pointwise displacement field. ${ }^{8}$ In contrast, global DIC simultaneously tracks the motion of all pixels within the region of interest, resulting in the measurement of motion of a complete mesh. ${ }^{9}$ This mesh allows for convenient comparison or coupling with finite element analysis ${ }^{10}$ and ensures that continuity constraints are maintained, leading to more accurate measurements. ${ }^{11}$ In both local and global DIC, the derivatives of the calculated displacement fields are used to compute the strains. In this paper, local DIC is used because of advantages of ease of use and computational efficiency, ${ }^{12}$ and it should be assumed that future uses of the phrase "DIC" refer to the local DIC technique.

For the correlation algorithm to capture the motion, DIC requires a high-contrast speckle pattern on the specimen surface. ${ }^{13}$ The size of the speckles in the applied pattern must ensure that 
each subset has a unique array of grayscale values and pixel arrangements. ${ }^{14}$ If the speckle is too small for the length scale of a test, the subset will become a collection of similar-valued pixels. If it is too large, there is a greater risk of an entire subset being dominated by a single speckle, rendering different subsets indistinguishable. Thus, the subset size used in the correlation depends on the speckle pattern applied. A smaller subset and smaller spacing between subsets result in more data points compared with a larger subset, but smaller subsets are also more susceptible to noise which affects the uncertainty in the measurement. ${ }^{15}$ Choosing an appropriate subset size has a great impact on the accuracy of the calculated displacements, and the proper subset size will depend on the individual speckle pattern. ${ }^{16}$

At extreme temperatures, there are additional challenges to performing DIC. Above $600^{\circ} \mathrm{C}$, materials emit Planck radiation within the visible spectrum which can saturate the camera sensor. ${ }^{17}$ The emitted light is known to be brighter at longer wavelengths, and so taking images primarily with shorter-wavelength blue light sources and filters has been shown to effectively raise the temperature at which images useful for DIC can be recorded. ${ }^{18}$ By eliminating longer wavelengths of light using an optical bandpass filter, the light emitted from the heated specimen does not oversaturate the image captured by the camera. This has been well-demonstrated using blue lights, filters, and optics up to temperatures ranging from $1000{ }^{\circ} \mathrm{C}$ for crack-opening displacement measurements ${ }^{19}$ to $1500{ }^{\circ} \mathrm{C}$ for ceramic composites, ${ }^{20}$ with other notable work in that temperature range made possible with blue light. ${ }^{21,22}$

Evidence suggests that the temperature range can be extended further with the use of ultraviolet (UV) light sources and filters, utilizing even shorter wavelengths. ${ }^{23}$ At a given temperature, UV light is emitted from the specimen at a lower intensity than light with longer wavelengths, including blue. However, this method requires that all lenses transmit light with shorter wavelengths which are allowed to pass through the filter, and that the camera sensor is sensitive to those shorter wavelengths. Additional light sources that provide incident light at the desired wavelength are also necessary. UV-DIC has been demonstrated at the centimeter scale up to at least $1600{ }^{\circ} \mathrm{C}$ using commercial UV lenses and can potentially be used even higher on materials with higher melting points. ${ }^{24}$ However, there is currently no commercially available high-magnification lens that will allow images to be taken (i) in the UV range, (ii) at a submillimeter scale, and (iii) at a long enough working distance for a specimen to be placed inside a testing chamber while the camera is placed outside the chamber window.

In this work, a custom high-magnification UV lens is presented to perform DIC. The lens is demonstrated using a series of thermo-mechanical experiments on a steel ring specimen. A hightemperature test, a rigid body motion test, and a mechanical deformation test were conducted. For each of the three tests, a two-camera setup was used to compare the custom high-magnification UV lens with a commercially available UV lens.

\section{MATERIALS AND METHODS}

\section{A. Custom high-magnification UV lens}

To extend UV-DIC measurements to the submillimeter range, a custom high-magnification UV lens was designed to resolve a $2.2 \mathrm{~mm}$ horizontal field of view at a $254 \mathrm{~mm}$ working distance. Furthermore, the design was restricted to using off-the-shelf components to reduce costs and allow in-house assembly. To meet the above criteria, an infinity-corrected, telephoto lens with a nominal 3.2 paraxial magnification was designed as follows.

The custom UV lens assembly was designed to be paired with a JAI CM-140GE-UV camera with C-mount type threading. The camera features a $6.4 \mathrm{~mm}$ sensor with a sensor pixel size of $4.65 \mu \mathrm{m}$. When paired with a UV lens, the camera produces images which contain $1392 \times 1040$ pixels. Thus, when the horizontal field of view of $2.2 \mathrm{~mm}$, each pixel represents an area roughly $1.60 \mu \mathrm{m}$ square. The assembly can be paired with a UV bandpass filter developed by Llewellyn Data Processing (LDP) LLC, and a pair of external UV light sources with a peak wavelength of $365 \mathrm{~nm}$. This pairing of equipment has been demonstrated in previous experiments to successfully perform UV-DIC. ${ }^{2}$

The lens was designed with the commercial software package OpticStudio, developed by Zemax LLC for designing and characterizing optical assemblies. A ray diagram of the lens is shown in Fig. 1(a). The entire assembly was built in-house using off-the-shelf

a)

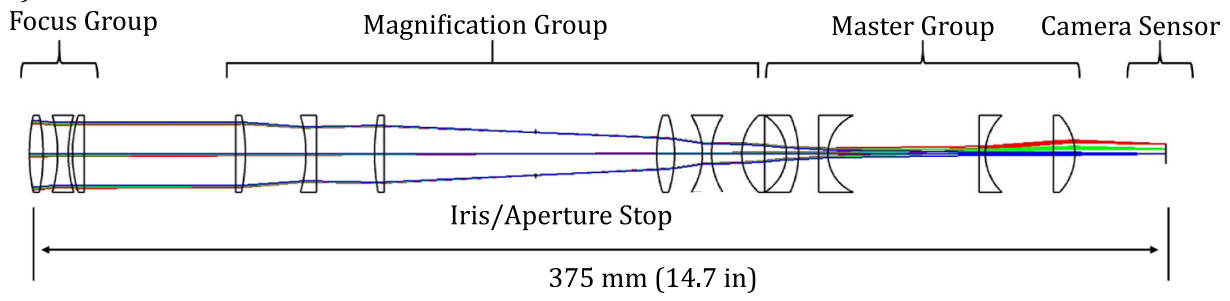

b)

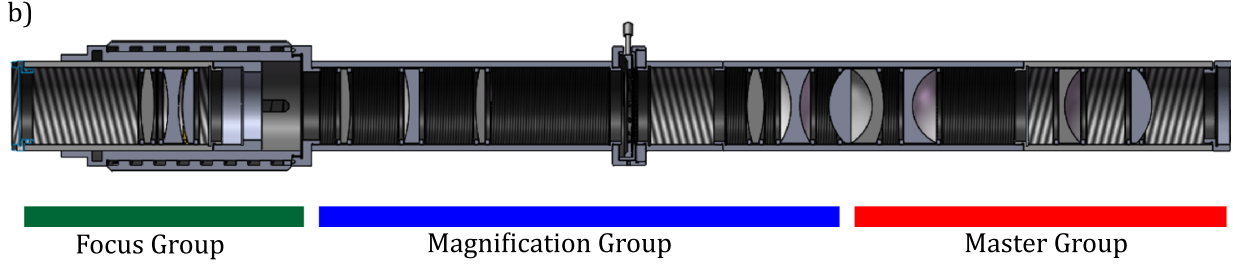

FIG. 1. (a) Ray diagram of the 13 lenses used in the assembly, with the three lens groups indicated. (b) Crosssectional view of the custom UV lens assembly and group subassemblies. 
components, which were bought primarily from Edmund Optics and ThorLabs, Inc., including 13 singlet lenses. The lenses can be separated into three functional groups: a translating focus group, a stationary magnification group, and a stationary master group, seen in the cross section of the assembly, shown in Fig. 1(b).

The focus group consists of three lenses (LB5247-E, LD4293UV, and LA5817-E, from ThorLabs) having an effective focal length equivalent to the desired working distance, collecting light as it enters the assembly and collimating it to be used by the magnification group. $^{25,26}$ These lenses are placed in a telescoping lens tube (SM1NR1, ThorLabs). This technique is used in infinity-corrected microscopes and allows fine focal point adjustments to be made after positioning the camera.

The magnification group receives the collimated light from the focus group and extends the focal point to the master group, ${ }^{27}$ magnifying the image by a factor of $3.2 \times$. The group of lenses (two of LA5012-E, and one each of LC4888-UV, LB5284-E, LD4931-UV, and LA5370-E from ThorLabs) is placed in a static housing (parts SM1L40 and SM1L10, ThorLabs) resulting in a fixed magnification power. An adjustable iris (SM1D25, ThorLabs) is used to change the effective f-number of the system, thereby adjusting the diffraction limit, spot size, and depth of field. ${ }^{28}$ The diameter of the selected iris ranges from 5 to $20 \mathrm{~mm}$.

The purpose of the master group is to receive light from the magnification group to correct aberrations ${ }^{27}$ and transmit the image to the camera sensor. The group is designed as a telephotolens system, shortening the effective focal length of the assembly, and consists of four lenses (LF5895-E, LC4252-UV, LC5269-E, and LA4306-UV, ThorLabs). This allows closer placement of the camera sensor to the lenses. The master group is placed in another static housing (SM1L40 and modified part SM1L25-MOD of depth $58.01 \mathrm{~mm}$ ), separate from the magnification group and features a C-mount adapter (SM1A975, ThorLabs) to connect the overall assembly to the camera. Additional information on parts used and assembly are referenced in the supplementary material section.

In order to reduce chromatic aberrations, the lenses used throughout the assembly alternate between calcium fluoride and fused silica, both of which are known to be good transmitters of UV light, with a transmittance between 0.7 and 0.9 through most of the ultraviolet range. ${ }^{29}$ The assembly is fully color-corrected for wavelengths between 270 and $400 \mathrm{~nm}$. Due to the relatively large number of lenses in the assembly, the overall transmissivity is $58 \%$ at the
$365 \mathrm{~nm}$ wavelength, which corresponds to the peak wavelength of the external light sources.

The entire assembly has an overall working distance of $254 \mathrm{~mm}$, allowing the camera and lens to be placed safely outside an environmental chamber while focusing through a window to a test specimen inside. This is critical for use with the intended high-temperature testing. When focused at such a distance, the camera and lens have a field of view of about $2.2 \times 1.7 \mathrm{~mm}$. To evaluate the above design, the camera and lens were aimed at a USAF 1951 test target and a series of Ronchi rulings. The maximum resolution was found to resolve to between 101.6 and 110 line pairs $/ \mathrm{mm}$.

\section{B. Thermo-mechanical experiments}

Three thermo-mechanical experiments were performed: (i) a rigid body motion test to compare DIC measurements made with the custom lens with known displacements, (ii) a thermal test to capture high-temperature images, and (iii) a mechanical deformation test to demonstrate the ability for the custom lens to measure strain.

In each demonstration of the designed lens, the specimen used was a T-316 stainless steel ring, approximately $12.7 \mathrm{~mm}$ in diameter, $3 \mathrm{~mm}$ in width, and with a wall thickness of $1.2 \mathrm{~mm}$, similar to the dimensions of some Light Water Reactor (LWR) zirconium fuel cladding testing samples. The high-magnification lens, focused on a single section of the thickness of the ring, was used to capture images at the same time as a camera with a commercially available, lowmagnification lens, which captured images of the entire ring. The low-magnification lens used was a UKA Optics UV5035B $50 \mathrm{~mm}$ focal-length lens from Universe Kogaku America. This two-camera setup, used in all three phases of testing, allows for direct comparison of the custom high-magnification lens with its commercially available low-magnification counterpart.

A high-contrast pattern was applied to the top surface of the ring with Very High Temperature (VHT) flameproof spray-paint, which is rated for temperatures up to $1090{ }^{\circ} \mathrm{C}$ and is commonly used to perform high-temperature DIC. ${ }^{30}$ The black speckles were applied with an airbrush by making a fine mist of slightly thinned paint above the surface, letting the mist droplets settle onto the white surface. This resulted in a very small, high-contrast, black-speckle pattern on a white background appropriate for both the larger and the smaller field of view. The speckle size varied, but the smallest speckles were roughly $10-20 \mu \mathrm{m}$ across as shown in Fig. 2 .
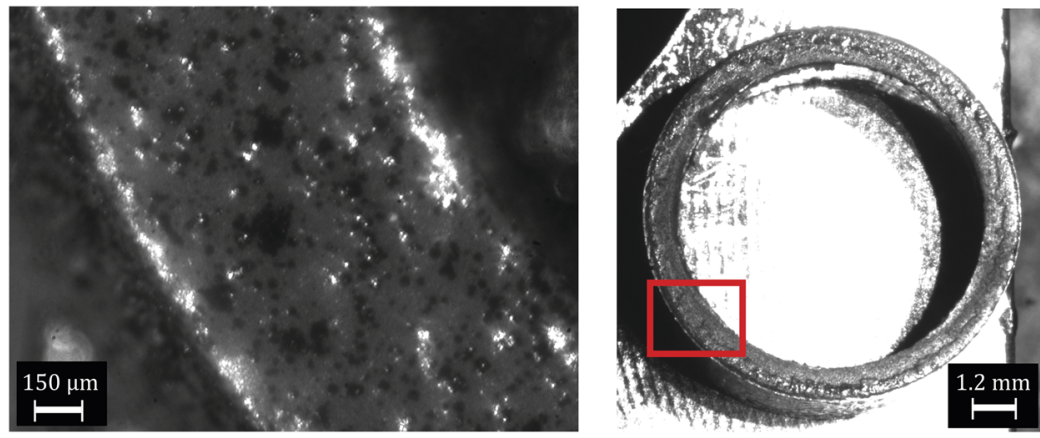

FIG. 2. Sample Speckle Pattern, taken with highmagnification lens ("left") and low-magnification lens ("right"). The high-magnification image has been brightened for legibility by multiplying pixel values by a uniform factor of 2 using MATLAB. 


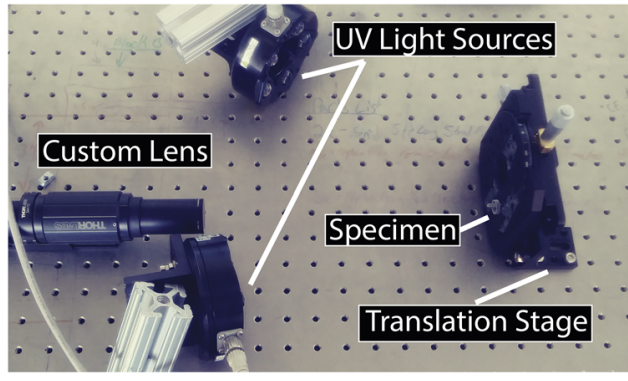

FIG. 3. Rigid body motion experimental setup with lenses (left), UV external light sources (top center, bottom center), and translating stage with specimen (right).

\section{Rigid body motion test}

The specimen was mounted to a micrometer-driven stage made by Thor Labs, Inc. The stage applied rigid translations in $0.0254 \mathrm{~mm}$ intervals in both the vertical and horizontal directions. The stage and camera assembly were rigidly fixed to an optical table, to minimize any relative displacement between the specimen and the cameras, aside from the applied displacements. The cameras were positioned to be perpendicular to the stage so the translations applied to the specimen would result in predominantly in-plane motion.
The custom lens was placed $254 \mathrm{~mm}$ away from the specimen and the $50 \mathrm{~mm}$ lens was placed $330 \mathrm{~mm}$ away. The rigid body motion experimental setup is shown in Fig. 3.

Reference images of the specimen surface were collected with the high-magnification lens and camera prior to any motion. Displacements were then applied in both the positive and negative horizontal directions at 8 regular increments, resulting in displacements ranging from -0.203 to $0.203 \mathrm{~mm}$, relative to the position of the reference image. Images were taken after each translation. Motion was small to ensure that the region of interest for the custom lens remained in the field of view in all images. The process was then repeated for the vertical direction with the same camera. The lowmagnification lens and camera then replaced the other lens and camera at the position perpendicular to the specimen, and the same method was followed to capture the horizontal and then vertical translations.

\section{Thermal test}

For the thermal test, the specimen was tested in a temperaturecontrolled load frame chamber, the Gleeble 1500D, shown in Fig. 4(a). The system consists of a load frame and resistive heater inside an environmental test chamber, which can support air, vacuum, or inert gas environments. The chamber features a borosilicate glass window, allowing images to be taken for DIC. Specimens

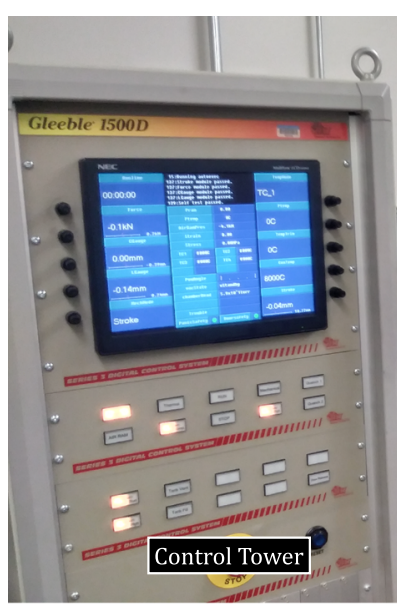

b)

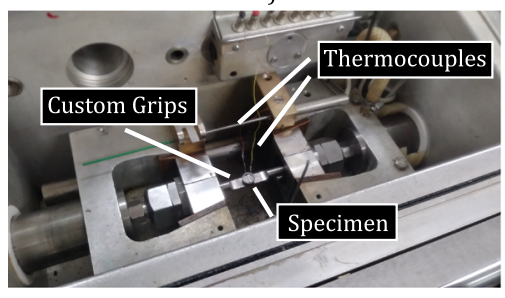

a)

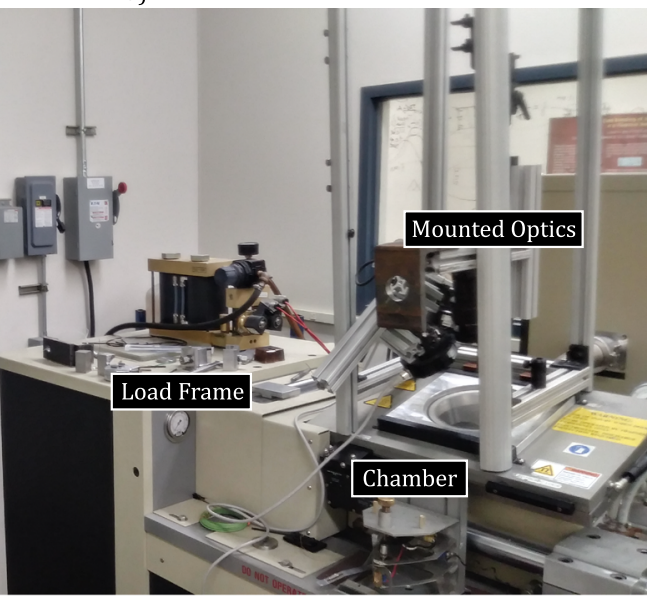

c)

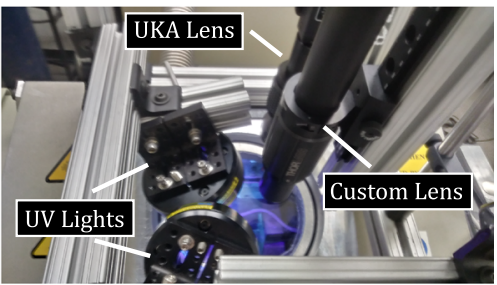

FIG. 4. (a) Gleeble $1500 \mathrm{D}$ control tower, load frame and testing chamber, and mounted cameras and light source; (b) ring specimen with K-type thermocouples loaded into custom hooked grips in the Gleeble 1500D standard specimen grips; (c) mounted optics, with UKA Optics $50 \mathrm{~mm}$ lens and camera, top middle, custom lens, middle right, and UV light source, bottom left, over the environmental chamber viewing window; (d) diagram of custom hooked grip with (A) threaded end, (B) flat ring resting surface, and (C) semicircle grip.

d)

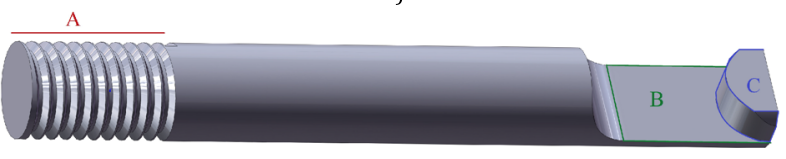


are heated by passing an electrical current through the water-cooled grips to an electrically conductive specimen, reaching the desired temperature via joule heating. Temperatures of at least $1600{ }^{\circ} \mathrm{C}$ have been achieved with the Gleeble to demonstrate DIC, ${ }^{24}$ and the machine is rated for use to $3000{ }^{\circ} \mathrm{C}$. For these thermal and mechanical tests, the ring specimen was placed in custom grips located within the environmental chamber, shown in detail through the chamber window in Fig. 4(b).

The custom lens had the aperture opened as wide as possible to allow enough light in the image, and so the adjustable iris diameter was $20 \mathrm{~mm}$. The UKA $50 \mathrm{~mm}$ lens had the aperture opened to give an effective f-number of 16 . The front of the custom lens was placed $254 \mathrm{~mm}$ away from the specimen and the front of the $50 \mathrm{~mm}$ lens was placed $330 \mathrm{~mm}$ away from the specimen. Each of the cameras and the UV light source were mounted using aluminum T-slot frames and were arranged so that the cameras were perpendicular to the specimen surface. The optical system, mounted on top of the chamber window, is shown in Fig. 4(c).

The specimen was supported by a pair of hooked grips, made from T-303 stainless-steel rods with a diameter of $10 \mathrm{~mm}$, shown in Fig. 4(d). One end of the grip is threaded [labeled (A) in the figure] to accommodate a backing nut which is pulled to apply tension. The hook features a flat surface [labeled (B)] on which the specimen rests. At the right end of the grip is a semicircle-shaped hook [labeled (C)], which goes on the inside of the ring. Each grip is fastened into the Gleeble and the ring is placed over the two semicircles. Once loading begins, the inside face of the ring comes into contact with the curved face of the semicircle hook (C), and the bottom face of the ring remains in contact with the flat face of the grip (B). The radius of curvature of the semicircle hook is slightly smaller than the inner radius of the ring, meaning that initially the two surfaces will contact only at one line on the circumference of the ring. The ring is then pre-loaded to $1.5 \mathrm{kN}$, which causes the ring to become noncircular as some deformation occurs and the ring begins to appear oblong. This is necessary for the specimen to fit the shape of the hook, increasing the contact area through which electrical current is applied for heating and preventing electrical arcing.

Before the thermal test, K-type thermocouples were welded to the outside of the ring specimen, centered between both the top and bottom surfaces, to provide temperature feedback to the thermal control system of the Gleeble. The specimen was oriented so the top flat surface of the ring was parallel to the camera. Reference images were taken after pre-loading but prior to heating. A thermal load was then gradually applied, raising the temperature to $900{ }^{\circ} \mathrm{C}$ by increments of $50{ }^{\circ} \mathrm{C}$ every $2 \mathrm{~min}$. At each increment, each of the cameras took an image. The grips were moved as the specimen was heated to allow for movement due to thermal expansion without losing electrical contact, while keeping the load at or below $1.5 \mathrm{kN}$ to avoid excessive permanent deformation. It should be noted that as temperatures increased, the ring slowly continued to deform and elongate as the load necessary for electrical contact was maintained. Upon completion of the test, the temperature was decreased to room temperature, the load was released, and the specimen was removed.

\section{Mechanical deformation test}

The specimen was loaded in the same grips and with the same method as described in the thermal test. The camera arrangements and orientations were also similar, although the aperture was reduced from a diameter of $20 \mathrm{~mm}$ to $10 \mathrm{~mm}$ to improve depth of field, and a second UV light source was used to compensate for the reduced light transmission through the smaller aperture.

The specimen was deformed in displacement control while monitoring the resulting load reported by the load frame. A preload of $0.9 \mathrm{kN}$ was applied before any images were taken. This preload allowed the ring to become seated on the grips, ensuring contact between the grips and the ring to prevent slipping or translation during further loading. A reference image of the specimen was taken at the initial preloaded position using both the camera with the highmagnification lens, and the camera with the commercial lens. The displacement gauge was then zeroed, and the grips were moved apart under displacement control at increments of $0.02-0.05 \mathrm{~mm}$ (smallest increment of displacement between grips) until a maximum displacement of $0.34 \mathrm{~mm}$. Subsequent images were captured with both cameras at each interval as the specimen was deformed. After reaching $0.34 \mathrm{~mm}$ grip displacement and capturing both images, the specimen was unloaded. Final images were taken of the unloaded specimen.

For comparison purposes, a finite element analysis was used to model the non-uniform deformation experienced by the ring specimen. The model was performed using FEMAP, a commercial finite element package from Siemens Corporation. Models of the ring and a simplified grip (a semicircle pin of the same radius as the semicircle grip used in the experiments) were produced, using quarter symmetry to simplify the analysis. The ring and grip were both meshed using solid hex elements. Contact between the ring and grip was modeled using FEMAP's built-in contact algorithms, and the load conditions were set to simulate those present in one of the images. NX Nastran advanced nonlinear static analysis was used and strains in the direction of loading were displayed on the contour of the deformed specimen. These strain contours serve as a coarse validation of strain distributions measured through DIC.

\section{Image processing}

DIC was performed on the images from the rigid body motion and mechanical deformation tests to determine the full-field displacements. The reference and displaced images were imported in VIC-2D, a commercial DIC software package by Correlated Solutions, LLC and then correlated. Several different subsets and step sizes were explored, ranging from subsets of 25 pixels to 57 pixels for the custom lens and 7 to 25 pixels for the commercial lens. Of the sizes explored, the combinations which produced the best spatial resolution and fewest dropped subsets are summarized in Table I. The VIC-2D software was also used to compute the full-field strains from the resulting displacements, using Lagrangian strain formulations.

TABLE I. Subset and step sizes used in VIC-2D.

\begin{tabular}{llcc}
\hline \hline Lens & \multicolumn{1}{c}{ Test type } & Subset size & Step size \\
\hline Custom lens & Rigid body motion & 31 pixels & 12 pixels \\
& Mechanical deformation & 31 pixels & 12 pixels \\
Commercial lens & Rigid body motion & 11 pixels & 5 pixels \\
& Mechanical deformation & 11 pixels & 5 pixels \\
\hline
\end{tabular}



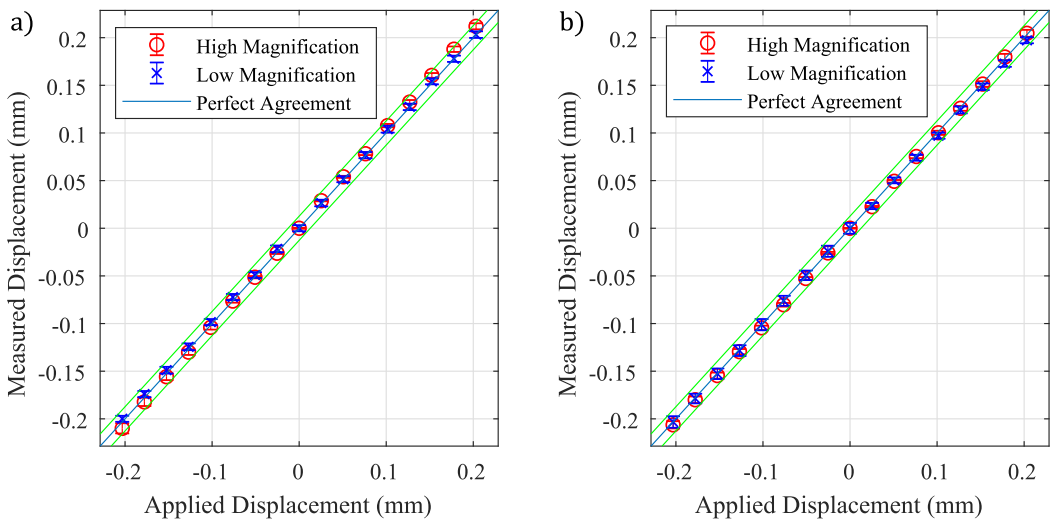

FIG. 5. Horizontal (a) and vertical (b) applied displacement for high- and low-magnification images.

\section{RESULTS}

\section{A. Rigid body motion test results}

The vertical and horizontal displacement results were compared with the known applied displacements. Figure 5(a) shows the horizontal applied displacements and averages of the measured displacements of the subsets in each high-magnification picture and each low-magnification picture. A similar plot for vertical displacements is shown in Fig. 5(b).
Both plots include a light blue line at a $45^{\circ}$ angle, representing perfect agreement between applied and measured displacements. Uncertainty bands represent twice the standard deviation of the measured displacements across all subsets of the image. The noise floor, defined here as the variation between static images due to random noise, is represented by the uncertainty bands at zero displacement, with values of $0.48 \mu \mathrm{m}$ for high magnification and $3.20 \mu \mathrm{m}$ for low magnification. The green bounding lines above and below the blue line are the measurement resolution uncertainty of the a) Commercial Lens Vertical Displacement

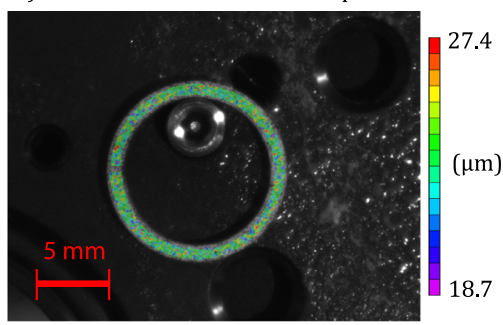

b) Custom Lens Vertical Displacement

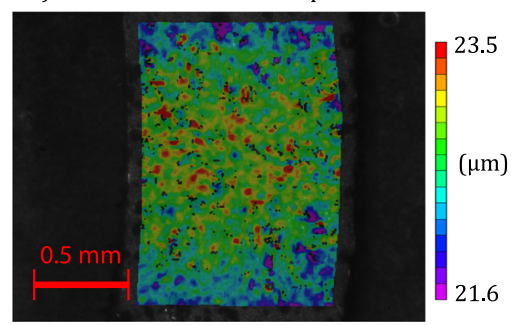

FIG. 6. Vertical displacement contour for (a) lowmagnification image, subset of 11 pixels, and (b) highmagnification image, subset of 31 pixels. a)

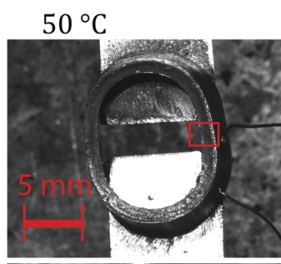

b)

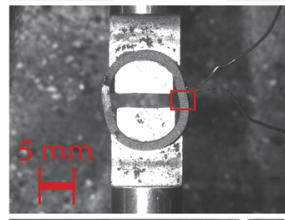

c)

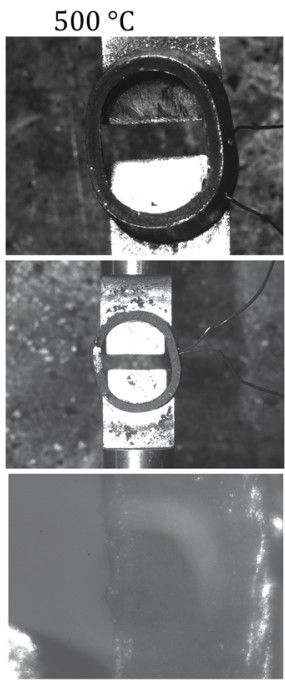

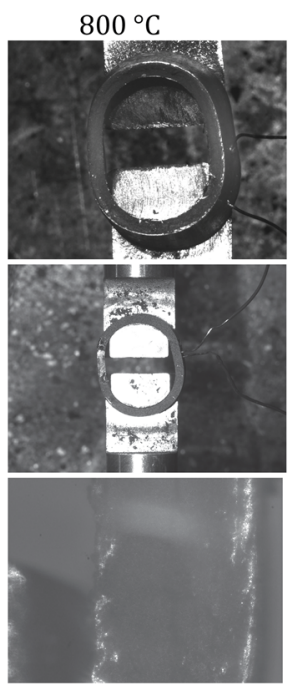

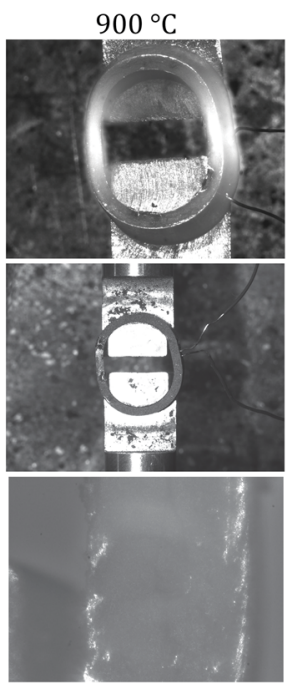

FIG. 7. Low-magnification without a UV bandpass filter (a), with a bandpass filter (b), and high-magnification (c) images at $50^{\circ} \mathrm{C}-900^{\circ} \mathrm{C}$. Red box shows placement of high-magnification image. 
a)

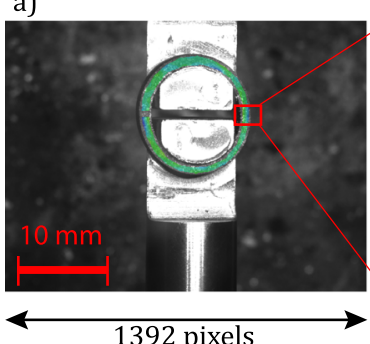

1392 pixels b)

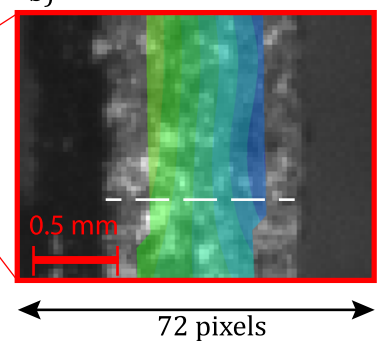

c)

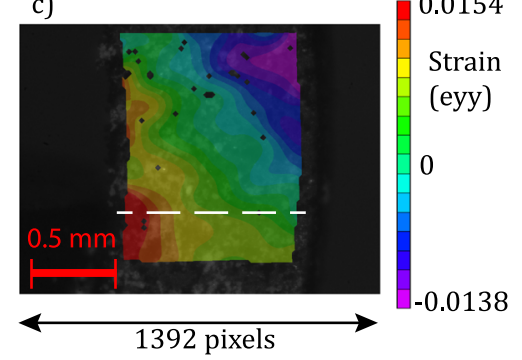

FIG. 8. Lagrangian full-field strain at $0.17 \mathrm{~mm}$ grip displacement (1.2 kN load) for (a) commercial lens (subset 11, step size 3), (b) zoomed in perspective of commercial image in (a), and (c) custom lens (subset 51 , step size 12). A white dotted line shows the location where data in Fig. 9 is sampled from both images. translation stage, indicating half a tick mark on the physical scale. The low-magnification images gave data comparable to the highmagnification images - for both, the reported displacements are very close to the applied displacements represented by the blue line and are within the green resolution uncertainty bounding lines.

A contour for the vertical displacement of the low-magnification image is shown in Fig. 6(a), while a contour of the highmagnification image at the same displacement is given in Fig. 6(b). It can be noted from the colored scale bars that both cover similar displacements. This suggests that the commercial lens and custom lens were both able to detect small displacements with similar success.

\section{B. Thermal test results}

Images were successfully captured at $50{ }^{\circ} \mathrm{C}$ increments using 3 cases: (a) the commercial $50 \mathrm{~mm}$ lens without a UV bandpass filter, (b) the commercial lens in conjunction with the filter, and (c) the custom lens which includes a built-in filter. Figure 7 shows the results for $50{ }^{\circ} \mathrm{C}, 500{ }^{\circ} \mathrm{C}, 800{ }^{\circ} \mathrm{C}$, and $900{ }^{\circ} \mathrm{C}$, respectively, for each of these cases. Note that the custom lens is focused on the region of the ring marked by the red box in both of the commercial lens

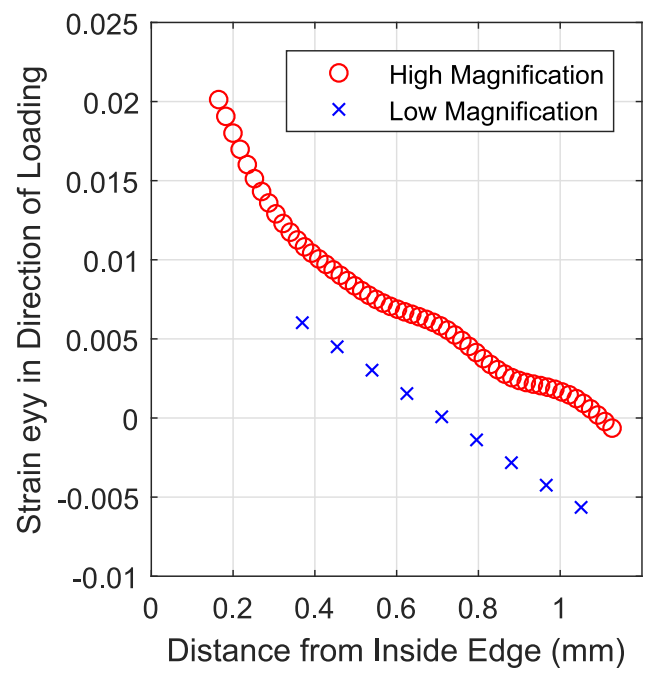

FIG. 9. Strain eyy through midline of ring as a function of wall thickness position, at grip displacement $0.17 \mathrm{~mm}$. images at $50{ }^{\circ} \mathrm{C}$. For clarity in this paper, the high-magnification images in Fig. 7 (c) have been artificially brightened by multiplying each pixel value by a uniform factor of 2.5 using MATLAB. However, the actual images used for processing in the DIC software were not brightened. As temperatures increase, significant glowing can be seen in the unfiltered, low-magnification images, while only slight, uniform changes appear in both of the other sets of images (b) and (c).

\section{Mechanical deformation test results}

The images of deformed states were produced by both lenses and imported into the DIC software as described previously. Contours showing full-field normal strain in the direction of loading at a representative load about midway through the test are shown in Fig. 8. Part (a) is the commercial lens contour; part (b) is the portion of the DIC contour in (a), artificially zoomed to be the size of the custom lens image; and part (c) is the custom lens contour. The noise floor for this strain measurement, interpreted as two standard deviations of the strain variation between repeated static images (similar to the noise floor described earlier with the rigid body motion), was 0.00078 for high magnification and 0.00153 for low magnification.

Both the high-magnification and low-magnification contours showed the greatest positive strain values at the inside edge of the

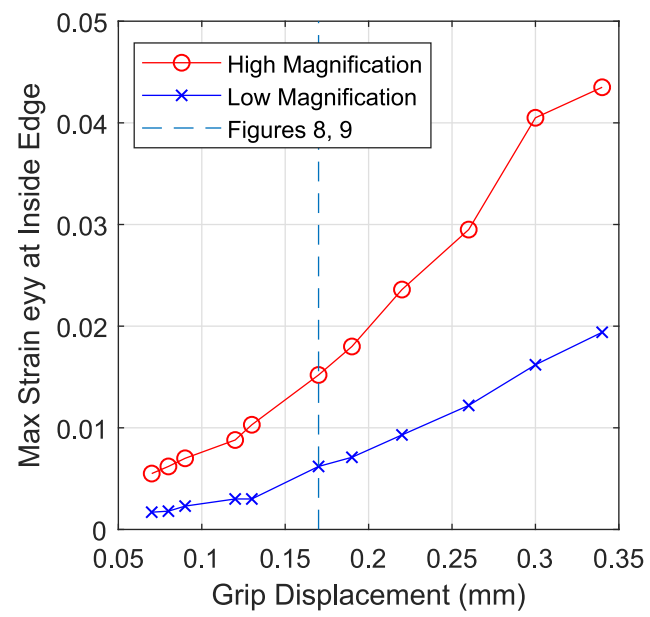

FIG. 10. Maximum positive strain in the direction of loading for high-magnification and low-magnification DIC contours. The dashed line indicates grip displacement corresponding with data presented in Figs. 8 and 9 . 
a)

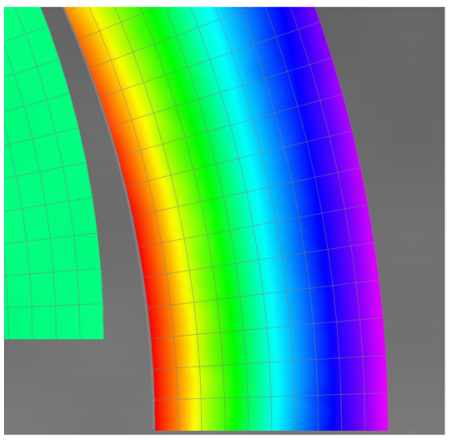

b)

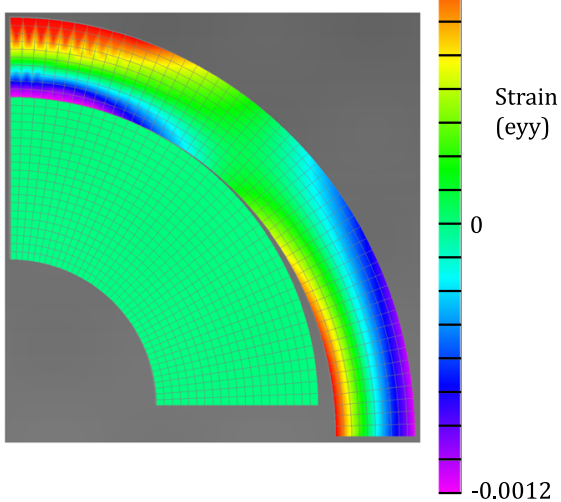

0.0012

FIG. 11. Finite element analysis loading-direction strain (eyy) contours, shown in (a) high magnification and (b) low magnification perspectives at a load of $1.2 \mathrm{kN}$ (equivalent to $0.17 \mathrm{~mm}$ grip displacement). Quarter symmetry has been used to simplify the model. ring, and the greatest negative strain values at the outer edge of the ring. This can be seen in Fig. 9, where the strain in the direction of loading is shown as a function of distance from the inside edge of the ring along a horizontal midline. The midline is placed at the same vertical location on the ring for both the high-magnification and low-magnification images. Note that because VIC-2D contours plot their data at the centers of subsets, each contour in Fig. 8 appears to be missing about a half-subset's worth of data along the perimeter of the region of interest. Thus, the data in Fig. 9 does not appear to span all the way to the inner edge of the ring, though the high magnification data is able to span closer to the edge than the low magnification data. However, they differ in the magnitude of those strains, as shown in Fig. 10. The high-magnification images produced by the custom lens led to consistently higher measured strains at the inside edge compared to the measurements resulting from the low-magnification images produced by the commercial lens.

The FEMAP model of the ring under the same load condition as Fig. 8 ( $1.2 \mathrm{kN}$ load, or $0.17 \mathrm{~mm}$ grip displacement) is shown in Fig. 11 in its deformed state, with the contour showing strain in the direction of loading. Both the high-magnification and low-magnification perspectives are shown.

\section{DISCUSSION}

The three tests demonstrate three key advantages of the custom lens over commercial UV lenses. First, the rigid body motion test demonstrated the ability to accurately measure a small, known, uniform displacement. Next, the thermal test demonstrated the ability for the custom lens (which includes a built-in UV band-pass filter) to record DIC images at high temperatures, while the unfiltered commercial lens produced saturated images at the same high temperatures. Finally, the deformation test demonstrated the ability to detect heterogeneous strains. Although both lenses agreed generally on the placement of high and low strain, they differ slightly in the degree of symmetry about the midline of the ring, and they differed significantly in terms of magnitude.

The rigid body motion test demonstrates the ability of the custom lens to produce accurate DIC results. Figure 5 shows that both lenses closely approach perfect agreement between measured and applied displacements. Inspection of the displacement contours shows that the high-magnification lens produces a smoother contour; that is, the displacement value of a subset is more likely to match its neighbors, when compared with the low-magnification contour. The success of the custom lens is at least partly due to the size and number of subsets that can be correlated. The custom lens allows for an increased number of pixels per $\mathrm{mm}$, which allows for more numerous and larger subsets. Conversely, the commercial lens allows many fewer pixels per mm, which limits both the number of subsets through the ring thickness and their size, leading to potential difficulties in correlation.

The high-temperature test demonstrated the ability of the custom lens to transmit UV light and therefore to avoid oversaturation of the image at temperatures that caused oversaturation in the commercial lens images. Comparing the images in Fig. 7 shows the difference that the custom lens assembly makes compared to a commercially available lens without a UV filter. At $50{ }^{\circ} \mathrm{C}$, the reference images show the speckled surface just above room temperature, where both camera setups produced usable images. At $500^{\circ} \mathrm{C}$, the figure shows the specimens at an elevated temperature, but still before glowing occurs. Compared to $50{ }^{\circ} \mathrm{C}$, the high-magnification image at $500{ }^{\circ} \mathrm{C}$ is slightly lighter and the low-magnification image shows some lightening as well. At $800^{\circ} \mathrm{C}$, a very faint glow is apparent at the bottom surface of the ring in the low-magnification image. This is where the specimen is beginning to heat up as it approaches a temperature where the visible light will emit with greater intensity. Finally, at $900{ }^{\circ} \mathrm{C}$, the unfiltered images show very significant saturation in the low-magnification image. The speckle contrast that was previously visible in the image is lost in the glow.

The upper limit of temperatures tested $\left(900^{\circ} \mathrm{C}\right)$ was limited by the operating temperatures of the test sample, not the capabilities of the lens. In principle, the lens should be able to record suitable DIC images at much higher temperatures given materials with higher melting points. In fact, UV-DIC has already been demonstrated to at least $1600{ }^{\circ} \mathrm{C}$ using commercial UV lenses, ${ }^{24}$ which the custom lens should be equally capable of reaching when paired with sufficiently bright light sources.

The mechanical deformation test showed that the custom lens can be implemented successfully into an experimental setup to detect heterogeneous strain via DIC. The working distance of 
$254 \mathrm{~mm}$ allows tests to be conducted on specimens inside environmental chambers, while the camera sits safely outside of a viewing window.

The mechanical deformation test also demonstrated the usefulness of the custom lens in capturing strain concentrations or features on a smaller scale. The low-magnification strain contour in Fig. 8 shows that at the inside edge of the ring, there may be a small region of higher positive strain. This is an expected result from the loading of the ring, which would cause a bending moment on the ring, as it acts like a curved beam being straightened. However, this result from the low-magnification is difficult to differentiate from noise in the measurement, and this contour alone may be inconclusive. The high-magnification contour confirms the strain distribution hinted at by the low-magnification measurement and shows a much more detailed strain distribution. Figures 8 (b) and 8(c) clearly shows the difference between the low- and high-magnification perspectives of the same area. The high-magnification contour is able to represent the strain field in greater detail because of the physical size represented by each subset and the increase in number of subsets being used.

The high-magnification strain field also gives a higher value for the maximum strain in the direction of loading in the ring, as seen in Fig. 10. At each applied displacement where an image was taken, the custom lens produced a peak strain measurement roughly twice that of the measurement produced by the commercial lens. This is expected for two reasons: (i) the higher magnification allows subsets to be used closer to the edge of the ring, and (ii) the higher magnification means each subset covers a smaller area. Because the highest strains occur at the edges of the ring, subsets closer to the edge will better capture the higher strains. In addition, the smaller area of an individual subset results in better spatial resolution. A subset strain value will be "averaged" across the entire subset, so a larger subset is not able to capture the extreme strain values as well as a smaller subset is able to capture them. These two phenomena of capturing strains closer to the edge of the ring, and of reducing the spatial averaging of the subset, are clearly shown in Fig. 9. The high-magnification clearly gets closer to both the inside and outside edges of the ring, and it is able to capture higher strains because of the smaller subset size. This demonstrates the advantage of the highmagnification lens holds in experiments where large spatial strain gradients are expected.

The finite element analysis confirms the strain distribution presented by both the high-magnification and low-magnification DIC results. Simplifying assumptions were made which may contribute to the inaccuracy of the strain magnitudes. However, the location of high positive and negative strains do match the results given by both the low- and high-magnification lens. This provides some confirmation that type of deformation is similar to straightening out a curved beam, resulting in tensile strain on the inside edge and compressive strain on the outside edge. Curved beam theory equations $^{31}$ predict a strain in the direction of loading of 0.0098 under the load conditions matching the $0.17 \mathrm{~mm}$ grip displacement and at the first distance from the edge which was captured by both high- and low-magnification in Fig. 9. This agrees well with the high-magnification measurement at the same location. These combined analyses demonstrate that the custom lens can effectively measure the mechanically induced deformations experienced by the specimen
One drawback of the custom lens is that it transmits relatively little light. With 13 singlet lenses in the assembly, the transmissivity is low and the resulting images appear less bright. This can be countered in an experiment by opening the aperture, but that can result in a less focused image due to decreased depth of field. Brightness can also be improved by increasing exposure time, but this can also lead to potential blurring of the image if the object is moving. ${ }^{32}$ This can be especially difficult in vacuum chambers if the vibration of a vacuum pump imposes relative motion between the camera and a test specimen. Thus, it is important to ensure that sufficient light reaches the specimen when the lens is used, especially in a dark environment such as a testing chamber.

It can be seen in the images taken with the custom lens that a slight "ghost ring" image appears, centered on the middle of the image, as shown against a white paper background in Fig. 12. Moving the image in and out of focus demonstrated that this aberration is more pronounced when the image is not in focus and is less visible as the focus of the image is improved. The results of the rigid body motion tests show that this does not necessarily interfere with the ability of DIC to measure the deformation. The results of these tests clearly demonstrate that images produced by a camera fitted with the custom lens can accurately measure the displacement of a specimen. When comparing the contour plots with the ghost ring placement in Fig. 6, vertical displacement contour for (a) low-magnification image, subset of 11 pixels, and (b) high-magnification image,
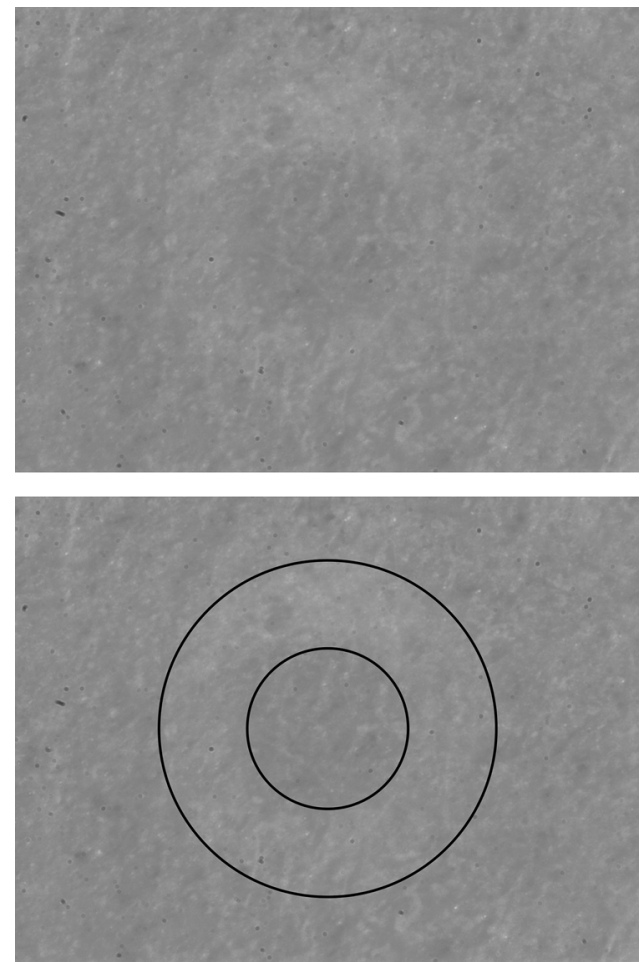

FIG. 12. ("top") Image of a white paper background, obtained using the custom UV lens; ("bottom") the same image, with dark lines emphasizing the boundary of the ghost ring. 
subset of 31 pixels, there is no association between the areas where the ring appears and the dropping of subsets in the image due to a poor correlation from the DIC software.

One of the most impressive capabilities offered by the custom lens is the improvement in the field of view despite a relatively long working distance. This can benefit many potential applications where small features are studied. When a low-magnification lens is used outside the chamber, the entire ring specimen is captured by a field of view $38.7 \mathrm{~mm}$ across, yet the majority of the image is not filled by the specimen. The difficulty that arises with this field of view comes from the thickness of the ring being viewed. From a comparable working distance, the field of view for the camera with the custom lens was $2.2 \mathrm{~mm}$ across, allowing the camera to focus on a smaller portion of the ring where higher strain values are present. The result of the custom lens is more displacement data over a smaller region, with each displacement measurement being more reliable when compared with the commercial lens.

Another advantage of the UV lens is that the diffraction limit of light is wavelength-dependent, and thus UV microscopy is known to achieve better resolutions compared to optical microscopy using light with longer wavelengths. Based on a wavelength of $365 \mathrm{~nm}$ and a working distance of $254 \mathrm{~mm}$, the Airy disc radius is estimated to be $15.34 \mu \mathrm{m}$ using software from Zemax. By contrast, blue light has a wavelength closer to $450 \mathrm{~nm}$, and thus its Airy disc is significantly larger. As the new lens has been shown to resolve Ronchi rulings down to 110 line pairs $/ \mathrm{mm}$, this means that the new lens can resolve down to the diffraction limit of UV light at its working distance.

More broadly, this lens has potential application in a wide range of fields other than solid mechanics. UV imaging has been used in the analysis of electrical components through detection of corona discharge, ${ }^{33}$ in vitro drug release testing, ${ }^{34}$ and visualization of latent fingerprints in forensic sciences. ${ }^{35}$ In addition, the shorter wavelength of UV light allows the detections of small surface features otherwise invisible, such as scratches. ${ }^{36}$ The long working distance and high magnification capabilities of this custom lens could easily lend it to use in these or other UV imaging applications, in addition to UV-DIC.

\section{CONCLUSIONS}

In summary, a custom UV lens was designed and demonstrated for high-magnification, high-temperature optical strain measurements. The custom lens was shown to meet three criteria: (i) It transmitted light in the UV range while blocking the high-intensity longer wavelengths through the use of a filter, while a traditional lens and camera without the filter did not; (ii) it was able to produce a field of view that was $2.32 \mathrm{~mm}$ across, a significant improvement over commercial UV lenses; and, (iii) it was demonstrated in an experimental setup with a working distance of $254 \mathrm{~mm}$, allowing it to be placed outside an environmental chamber window and to be protected from hazardous conditions.

The custom lens is capable of being used in future work where measurements of strain on a small region of interest, on the order of $2 \mathrm{~mm}$, are desired at high temperatures. This has potential application for material characterization of nuclear materials at accident conditions, ${ }^{37,38}$ studying microcrack fatigue or stress corrosion cracking, ${ }^{39}$ or other phenomena on a microscale at high temperatures. It can be beneficially implemented in these conditions to produce accurate, full-field strain, and displacement measurements on a small scale.

\section{SUPPLEMENTARY MATERIAL}

See supplementary material for complete drawings and bill of materials for the custom lens.

\section{ACKNOWLEDGMENTS}

This material is based upon work supported under an Integrated University Program Graduate Fellowship.

\section{REFERENCES}

${ }^{1}$ K. G. Field, M. N. Gussev, X. Hu, Y. Yamamoto, and R. H. Howard, "First annual progress report on radiation tolerance of controlled fusion welds in high temperature oxidation resistant FeCrAl alloys," U.S. Department of Energy, Office of Scientific and Technical Information, Oak Ridge National Laboratory (ORNL), Oak Ridge, TN, USA, 2015.

${ }^{2}$ F. Hild and S. Roux, Strain 42, 69 (2006).

${ }^{3}$ M. A. Sutton, J. J. Orteu, and H. Schreier, Image Correlation for Shape, Motion and Deformation Measurements: Basic Concepts, Theory and Applications (Springer Science \& Business Media, 2009).

${ }^{4}$ T. A. Berfield, J. K. Patel, R. G. Shimmin, P. V. Braun, J. Lambros, and N. R. Sottos, Exp. Mech. 47, 51 (2007).

${ }^{5}$ W. A. Scrivens, Y. Luo, M. A. Sutton, S. A. Collette, M. L. Myrick, P. Miney, P. E. Colavita, A. P. Reynolds, and X. Li, Exp. Mech. 47, 63 (2007).

${ }^{6}$ F. Di Gioacchino and J. Q. da Fonseca, Exp. Mech. 53, 743 (2013).

${ }^{7}$ J. D. Carroll, W. Abuzaid, J. Lambros, and H. Sehitoglu, Int. J. Fatigue 57, 140 (2013).

${ }^{8}$ R. Phillip, Exp. Tech. 36, 3 (2012).

${ }^{9}$ L. Wittevrongel, P. Lava, S. V. Lomov, and D. Debruyne, Exp. Mech. 55, 361 (2015).

${ }^{10}$ G. Besnard, F. Hild, and S. Roux, Exp. Mech. 46, 789 (2006).

${ }^{11}$ P. Cheng, M. A. Sutton, H. W. Schreier, and S. R. McNeill, Exp. Mech. 42, 344 (2002).

${ }^{12}$ B. Wang and B. Pan, Theor. Appl. Mech. Lett. 6, 200 (2016).

${ }^{13}$ T. Hua, H. Xie, S. Wang, Z. Hu, P. Chen, and Q. Zhang, Opt. Laser Technol. 43, 9 (2011).

${ }^{14}$ P. Reu, Exp. Tech. 39, 1 (2015).

${ }^{15}$ C. Lane, R. L. Burguete, and A. Shterenlikht, in Proceedings of the XIth Annual International Congress and Exposition (Society for Experimental Mechanics, 2008).

${ }^{16}$ B. Pan, H. Xie, Z. Wang, K. Qian, and Z. Wang, Opt. Express 16, 7037 (2008).

${ }^{17}$ B. Pan, D. Wu, Z. Wang, and Y. Xia, Meas. Sci. Technol. 22, 015701 (2011).

${ }^{18}$ P. Bing, X. Hui-min, H. Tao, and A. Asundi, Polym. Test. 28, 75 (2009).

${ }^{19}$ J. Blaber, B. S. Adair, and A. Antoniou, Rev. Sci. Instrum. 86, 035111 (2015).

${ }^{20}$ M. D. Novak and F. W. Zok, Rev. Sci. Instrum. 82, 115101 (2011).

${ }^{21}$ B. M. B. Grant, H. J. Stone, P. J. Withers, and M. Preuss, J. Strain Anal. Eng. Des. 44, 263 (2009).

${ }^{22}$ P. Leplay, O. Lafforgue, and F. Hild, J. Am. Ceram. Soc. 98, 2240 (2015).

${ }^{23}$ R. B. Berke and J. Lambros, Rev. Sci. Instrum. 85, 045121 (2014).

${ }^{24}$ T. Q. Thai, R. S. Hansen, A. J. Smith, J. Lambros, and R. B. Berke, "Importance of exposure time on DIC measurement uncertainty at extreme temperatures," Exp. Tech. (published online 2019).

${ }^{25}$ M. Laikin, Lens Design (CRC Press, 2006).

${ }^{26}$ E. B. Burgh, K. H. Nordsieck, H. A. Kobulnicky, T. B. Williams, D. O’Donoghue, M. P. Smith, and J. W. Percival, Instrument Design and Performance for Optical/Infrared Ground-Based Telescopes (International Society for Optics and Photonics, 2003), pp. 1463-1472. 
${ }^{27}$ R. Kingslake and R. B. Johnson, Lens Design Fundamentals (Academic Press, 2009).

${ }^{28}$ A. Davis and F. Kühnlenz, Opt. Photonik 2, 52 (2007).

${ }^{29}$ D. C. Harris, Infrared Phys. Technol. 39, 185 (1998).

${ }^{30}$ L. Rossmann, B. Sarley, J. Hernandez, P. Kenesei, J. Almer, J. Wischek, M. Bartsch, A. Köster, V. Maurel, and S. Raghavan, in 2018 AIAA/ASCE/AHS/ASC Structures, Structural Dynamics, and Materials Conference (American Institute of Aeronautics and Astronautics, 2018).

${ }^{31}$ A. P. Boresi, Advanced Mechanics of Materials, 6th ed. (Wiley, New York, 2002).

${ }^{32}$ C. Ma, Z. Liu, L. Tian, Q. Dai, and L. Waller, Opt. Lett. 40, 2281 (2015).
${ }^{33}$ E. G. da Costa, T. V. Ferreira, M. G. G. Neri, I. B. Queiroz, and A. D. Germano, IEEE Trans. Dielectr. Electr. Insul. 16, 985 (2009).

${ }^{34}$ J. Østergaard, E. Meng-Lund, S. W. Larsen, C. Larsen, K. Petersson, J. Lenke, and H. Jensen, Pharm. Res. 27, 2614 (2010).

${ }^{35}$ A. A. Cantu, Optics and Photonics for Counterterrorism and Crime Fighting III (International Society for Optics and Photonics, 2007), p. 67410D.

${ }^{36}$ M. Hasegawa and T. Shimakura, J. Appl. Phys. 107, 084107 (2010).

${ }^{37}$ T. Kubo, Y. Kobayashi, and H. Uchikoshi, J. Nucl. Mater. 435, 222 (2013).

${ }^{38}$ K. L. Murty and I. Charit, J. Nucl. Mater. 383, 189 (2008).

${ }^{39}$ J. A. Duff and T. J. Marrow, Corros. Sci. 68, 34 (2013). 